\title{
A Two-Phase Segmentation of Cell Nuclei Using Fast Level Set-Like Algorithms
}

\author{
Martin Maška ${ }^{1}$, Ondřej Daněk ${ }^{1}$, Carlos Ortiz-de-Solórzano ${ }^{2}$, \\ Arrate Muñoz-Barrutia ${ }^{2}$, Michal Kozubek ${ }^{1}$, and Ignacio Fernández García ${ }^{2}$ \\ 1 Centre for Biomedical Image Analysis, Faculty of Informatics \\ Masaryk University, Brno, Czech Republic \\ xmaska@fi.muni.cz \\ 2 Center for Applied Medical Research (CIMA) \\ University of Navarra, Pamplona, Spain
}

\begin{abstract}
An accurate localization of a cell nucleus boundary is inevitable for any further quantitative analysis of various subnuclear structures within the cell nucleus. In this paper, we present a novel approach to the cell nucleus segmentation in fluorescence microscope images exploiting the level set framework. The proposed method works in two phases. In the first phase, the image foreground is separated from the background using a fast level set-like algorithm by Nilsson and Heyden [1. A binary mask of isolated cell nuclei as well as their clusters is obtained as a result of the first phase. A fast topology-preserving level set-like algorithm by Maška and Matula 2] is applied in the second phase to delineate individual cell nuclei within the clusters. The potential of the new method is demonstrated on images of DAPI-stained nuclei of a lung cancer cell line A549 and promyelocytic leukemia cell line HL60.
\end{abstract}

\section{Introduction}

Accurate segmentation of cells and cell nuclei is crucial for the quantitative analyses of microscopic images. Measurements related to counting of cells and nuclei, their morphology and spatial organization, and also a distribution of various subcellular and subnuclear components can be performed, provided the boundary of individual cells and nuclei is known. The complexity of the segmentation task depends on several factors. In particular, the procedure of specimen preparation, the acquisition system setup, and the type of cells and their spatial arrangement influence the choice of the segmentation method to be applied.

The most commonly used cell nucleus segmentation algorithms are based on thresholding [34] and region-growing [56] approaches. Their main advantage consists in the automation of the entire segmentation process. However, these methods suffer from oversegmentation and undersegmentation, especially when the intensities of the nuclei vary spatially or when the boundaries contain weak edges.

Ortiz de Solórzano et al. 7] proposed a more robust approach exploiting the geodesic active contour model [8] for the segmentation of fluorescently labeled 
cell nuclei and membranes in two-dimensional images. The method needs one initial seed to be defined in each nucleus. The sensitivity to proper initialization and, in particular, the computational demands of the narrow band algorithm 9 severely limit the use of this method in unsupervised real-time applications. However, the research addressed to the application of partial differential equations (PDEs) to image segmentation has been extensive, popular, and rather successful in recent years. Several fast algorithms [10111] for the contour evolution were developed recently and might serve as an alternative to common cell nucleus segmentation algorithms.

The main motivation of this work is the need for a robust, as automatic as possible, and fast method for the segmentation of cell nuclei. Our input image data typically contains both isolated as well as touching nuclei with different average fluorescent intensities in a variable but often bright background. Furthermore, the intensities within the nuclei are significantly varying and their boundaries often contain holes and weak edges due to the non-uniformity of chromatin organization as well as abundant occurrence of nucleoli within the nuclei. Since the basic techniques, such as thresholding or region-growing, produce inaccurate results on this type of data, we present a novel approach to the cell nucleus segmentation in 2D fluorescence microscope images exploting the level set framework. The proposed method works in two phases. In the first phase, the image foreground is separated from the background using a fast level set-like algorithm by Nilsson and Heyden [1. A binary mask of isolated cell nuclei as well as their clusters is obtained as a result of the first phase. A fast topology-preserving level set-like algorithm by Maška and Matula [2] is applied in the second phase to delineate individual cell nuclei within the clusters. We demonstrate the potential of the new method on images of DAPI-stained nuclei of a lung cancer cell line A549 and promyelocytic leukemia cell line HL60.

The organization of the paper is as follows. Section 2 shortly reviews the basic principle of the level set framework. The properties of input image data are presented in Section 3. Section 4 describes our two-phase approach to the cell nucleus segmentation. Section 5 is devoted to experimental results of the proposed method. We conclude the paper with discussion and suggestions for future work in Section 6 .

\section{Level Set Framework}

This section is devoted to the level set framework. First, we briefly describe its basic principle, advantages, and also disadvantages. Second, a short review of fast approximations aimed at speeding up the basic framework is presented. Finally, we briefly discuss the topological flexibility of this framework.

Implicit active contours [12, have been developed as an alternative to parametric snakes 13. Their solution is usually carried out using the level set framework [14, where the contour is represented implicitly as the zero level set (also called interface) of a scalar, higher-dimensional function $\phi$. This representation has several advantages over the parametric one. In particular, it avoids 
parametrization problems, the topology of the contour is handled inherently, and the extension into higher dimensions is straightforward.

The contour evolution is governed by the following PDE:

$$
\phi_{t}+F|\nabla \phi|=0
$$

where $F$ is an appropriately chosen speed function that describes the motion of the interface in the normal direction. A basic PDE-based solution using an explicit finite difference scheme results in a significant computational burden limiting the use of this approach in near real-time applications.

Many approximations, aimed at speeding up the basic level set framework, have been proposed in last two decades. They can be divided into two groups. First, methods based on the additive operator splittings scheme 15 16] have emerged to decrease the time step restriction. Therefore, a considerable lower number of iterations has to be performed to obtain the final contour in contrast to standard explicit scheme. However, these methods require maintaining the level set function in the form of signed distance function that is computationally expensive. Second, since one is usually interested in the single isocontour the interface - in the context of image segmentation, other methods have been suggested to minimize the number of updates of the level set function $\phi$ in each iteration, or even to approximate the contour evolution in a different way. These include the narrow band [9], sparse-field [17], or fast marching method [10]. Another interesting approaches based on a pointwise scheduled propagation of the implicit contour can be found in the work by Deng and Tsui [18] or Nilsson and Heyden [1]. We also refer the reader to the work by Shi and Karl 11.

The topological flexibility of the evolving implicit contour is a great benefit since it allows to detect several objects simultaneously without any a priori knowledge. However, in some applications this flexibility is not desirable. For instance, when the topology of the final contour has to coincide with the known topology of the desired object (e.g. brain segmentation), or when the final shape must be homeomorphic to the initial one (e.g. segmentation of two touching nuclei starting with two separated contours, each labeling exactly one nucleus). Therefore, imposing topology-preserving constraints on evolving implicit contours is often more convenient than including additional postprocessing steps. We refer the reader to the work by Maška and Matula [2], and references therein for further details on this topic.

\section{Input Data}

The description and properties of two different image data sets that have been used for our experiments (see Sect. 5) are outlined in this section.

The first set consists of 10 images (16-bit grayscale, $1392 \times 1040 \times 40$ voxels) of DAPI-stained nuclei of a lung cancer cell line A549. The images were acquired using a conventional fluorescence microscope and deconvolved using the Maximum Likelihood Estimation algorithm provided by the Huygens software (Scientific Volume Imaging BV, Hilversum, The Netherlands). They typically contain both 

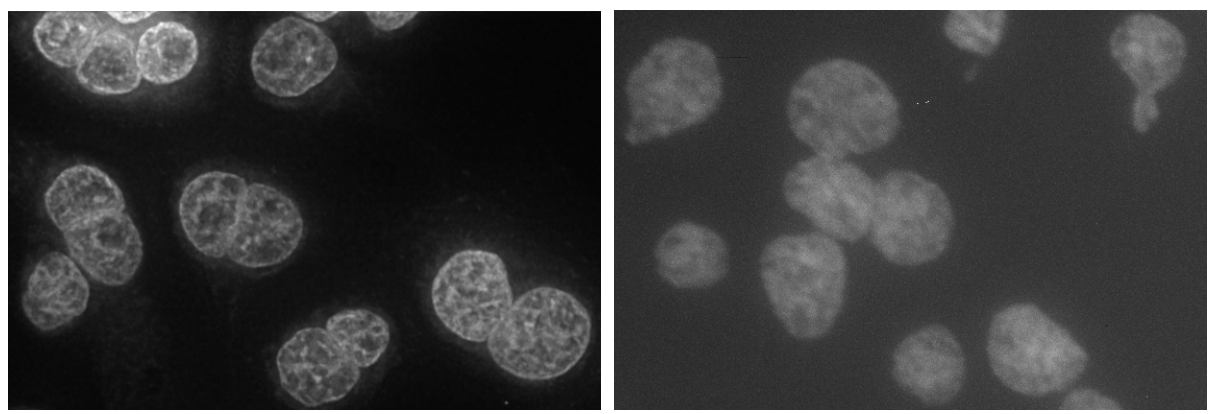

Fig. 1. Input image data. Left: An example of DAPI-stained nuclei of a lung cancer cell line A549. Right: An example of DAPI-stained nuclei of a promyelocytic leukemia cell line HL60.

isolated as well as touching, bright and dark nuclei with bright background in their surroundings originating from fluorescence coming from non-focal planes and from reflections of the light coming from the microscope glass slide surface. Furthermore, the intensities within the nuclei are significantly varying and their boundaries often contain holes and weak edges due to the non-uniformity of chromatin organization and abundant occurrence of nucleoli within the nuclei.

To demonstrate the potential of the proposed method (at least its second phase) on a different type of data, the second set consists of 40 images (8-bit grayscale, $1300 \times 1030 \times 60$ voxels $)$ of DAPI-stained nuclei of a promyelocytic leukemia cell line HL60. The images were acquired using a confocal fluorescence microscope and typically contain isolated as well as clustered nuclei with just slightly varying intensities within them.

Since we presently focus only on the 2D case, 2D images (Fig. 1) were obtained as maximal projections of the $3 \mathrm{D}$ ones to the $x y$ plane.

\section{Proposed Approach}

In this section, we describe the principle of our novel approach to cell nucleus segmentation. In order to cope better with the quality of input image data (see Sect. 3), the segmentation process is performed in two phases. In the first phase, the image foreground is separated from the background to obtain a binary mask of isolated nuclei and their clusters. The boundary of each nucleus within the previously identified clusters is found in the second phase.

\subsection{Background Segmentation}

The first phase is focused on separating the image foreground from the background. To achieve high-quality results during further analysis, we start with preprocessing of input image data. A white top-hat filter with a large circular structuring element is applied to eliminate bright background (Fig. 2a) in the 


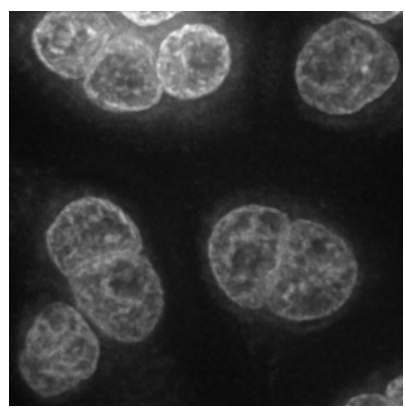

(a)

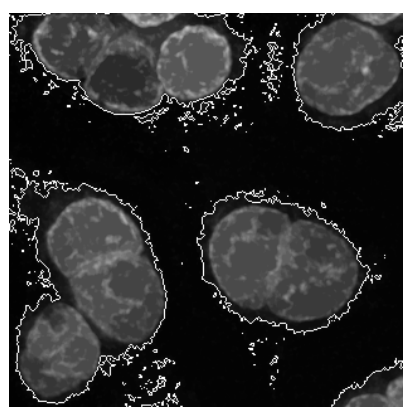

(d)

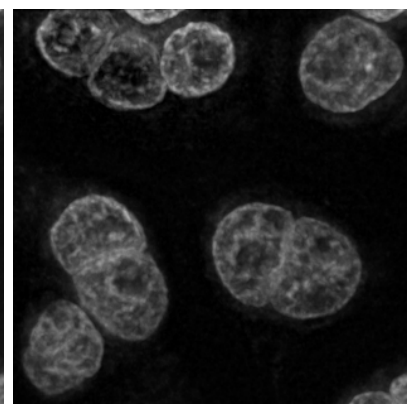

(b)

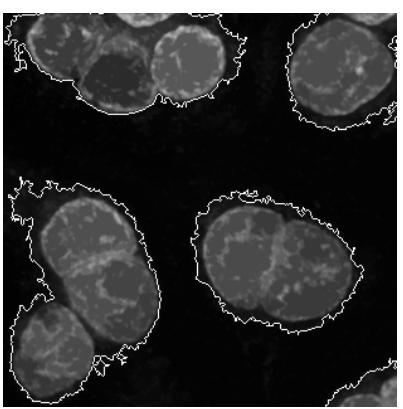

(e)

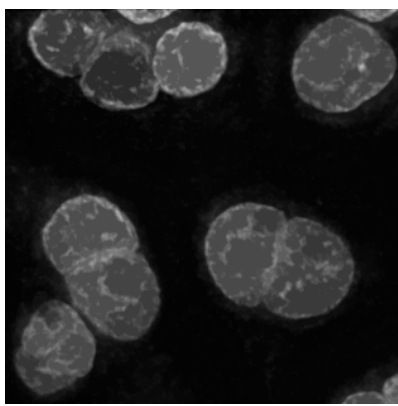

(c)

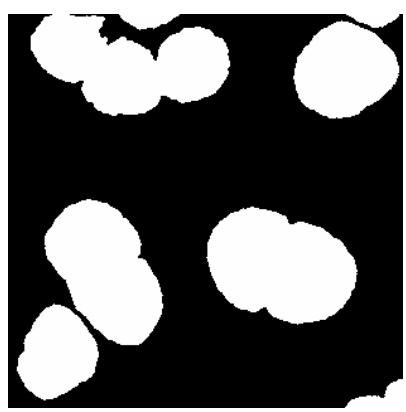

(f)

Fig. 2. Background segmentation. (a) An original image. (b) The result of a white top-hat filtering. (c) The result of a hole filling algorithm. (d) The initial interface defined as the boundary of foreground components obtained by applying the unimodal thresholding. (e) The initial interface when the small components are filtered out. (f) The final binary mask of the image foreground.

nucleus surroundings, as illustrated in Fig. 2b. Due to frequent inhomogeneity in the nucleus intensities, the white top-hat filtering might result in dark holes within the nuclei. This undesirable effect is reduced (Fig. 2k) by applying a hole filling algorithm based on a morphological reconstruction by erosion.

Segmentation of a preprocessed image $I$ is carried out using the level set framework. A solution of a PDE related to the geodesic active contour model [8] is exploited for this purpose. The speed function $F$ is defined as

$$
F=g_{I}(c+\varepsilon \kappa)+\beta \cdot \nabla P \cdot n .
$$

The function $g_{I}=\frac{1}{1+\left|\nabla G_{\sigma} * I\right|}$ is a strictly decreasing function that slows down the interface speed as it approaches edges in a smoothed version of $I$. The smoothing is performed by convolving the image $I$ with a Gaussian filter $G_{\sigma}(\sigma=1.3$, radius $r=3.0$ ). The constant $c$ corresponds to the inflation (deflation) force. The symbol $\kappa$ denotes the mean curvature that affects the interface smoothness. Its relative impact is determined by the constant $\varepsilon$. The last term $\beta \cdot \nabla P \cdot n$, where $P=\left|\nabla G_{\sigma} * I\right|, \beta$ is a constant, and the symbol $n$ denotes the normal to the interface, attracts the interface towards the edges in the smoothed version 
of $I$. We exploit the Nilsson and Heyden's algorithm [1], a fast approximation of the level set framework, for tracking the interface evolution.

To define an initial interface automatically, the boundary of foreground components, obtained by the unimodal thresholding, is used (Fig. 2 d). It is important to notice that not every component has to be taken into account. The small components enclosing foreign particles like dust or other inpurities can be filtered out (Fig. 22). The threshold

$$
\text { size }_{\min }=k \cdot \text { size }_{\text {avg }} \text {, }
$$

where $k \geq 1$ is a constant and size $e_{a v g}$ is an average component size (in pixels), ensures that only the largest components (denote them $S$ ) enclosing desired cell nuclei remain.

To prevent the interface from propagating inside a nucleus due to discontinuity of its boundary (see Fig. 3), we ommit the deflation force $(c=0)$ from (2).

Since the image data contains bright nuclei as well as dark ones, it is difficult to segment all the images accurately with the same value of $\beta$ and $\varepsilon$. Instead of setting these parameters properly for each particular image, we perform two runs of the Nilsson and Heyden's algorithm that differ only in the parameter $\varepsilon$. The parameter $\beta$ remains unchanged. In the first run, a low value of $\varepsilon$ is applied to detect dark nuclei. In the case of bright ones, the evolving interface might be attracted to a brighter background in their surroundings as its intensity is often similar to the intensity of dark nuclei. To overcome such problem, a high value of $\varepsilon$ is used in the second run to enforce the interface to pass through the brighter background (and obviously also through the dark nuclei) and detects the bright nuclei correctly. Finally, the results of both runs are combined together to obtain a binary mask $M$ of the image foreground, as illustrated in Fig. 2f.

The number of performed iterations is considered as a stopping criterion. In each run, we conduct the same number of iterations determined as

$$
N_{1}=k_{1} \cdot \sum_{s \in S} \operatorname{size}(s)
$$

where $k_{1}$ is a positive constant and size $(s)$ corresponds to the size (in pixels) of the component $s$.
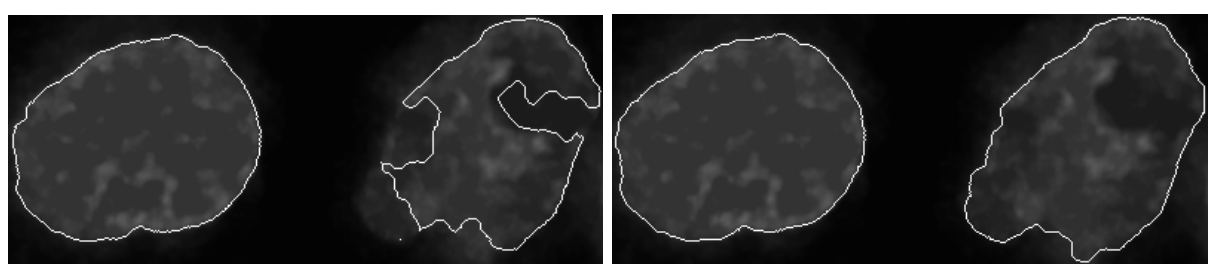

Fig. 3. The influence of the deflation force in (2). Left: The deflation force is applied $(c=-0.01)$. Right: The deflation force is omitted $(c=0)$. 


\subsection{Cluster Separation}

The second phase addresses the separation of touching nuclei detected in the first phase. The binary mask $M$ is considered as the computational domain in this phase. Each component $m$ of $M$ is considered as a cluster and processed separately. Since the image preprocessing step degrades significantly the information within the nuclei, the original image data is processed in this phase.

The number of nuclei within the cluster $m$ is computed first. A common approach based on finding peaks in a distance transform of $m$ using an extended maxima transformation is exploited for this purpose. The number of peaks is established as the number of nuclei within the cluster $m$. If $m$ contains just one peak (i.e. $m$ corresponds to an isolated nucleus), its processing is over. Otherwise, the cluster separation is performed.

The peaks are considered as an initial interface that is evolved using a fast topology-preserving level set-like algorithm [2]. This algorithm integrates the Nilsson and Heyden's one [1] with the simple point concept from digital geometry to prevent the initial interface from changing its topology. Starting with separated contours (each labeling a different nucleus within the cluster $m$ ), the topology-preserving constraint ensures that the number of contours remains unchanged during the deformation. Furthermore, the final shape of each contour corresponds to the boundary of the nucleus that it labeled at the beginning.

Similarly to the first phase, (11) with the speed function (2) governs the contour evolution. In order to propagate the interface over the high gradients within the nuclei, a low value of $\beta$ (approximately two orders of the magnitude lower than the value used in the first phase) has to be applied. As a consequence, the contour is stopped at the boundary of touching nuclei mainly due to the topologypreserving constraint. The use of a constant inflation force might, therefore, result in inaccurate segmentation results in the case of complex nucleus shape or when a smaller nucleus touches a larger one, as illustrated in Fig. 4. To overcome such complication, a position-dependent inflation force defined as a magnitude of the distance transform of $m$ is applied. This ensures that the closer to the nucleus boundary the interface is, the lower is the inflation force.

The number of performed iterations reflecting the size of the cluster $m$ :

$$
N_{2}=k_{2} \cdot \operatorname{size}(m),
$$

where $k_{2}$ is a positive constant, is considered again as a stopping criterion.

\section{Results and Discussion}

In this section, we present the results of the proposed method on both image data sets and discuss briefly the choice of parameters as well as its limitations. The experiments have been performed on a common workstation (Intel Core2 Duo 2.0 GHz, 2 GB RAM, Windows XP Professional).

The parameters $k, k_{1}, k_{2}, \beta$, and $\varepsilon$ were empirically set. Their values used in each phase are listed in Table 1. As expected, only $\beta$, which defines the sensitivity of the interface attraction force on the image gradient, had to be carefully set 

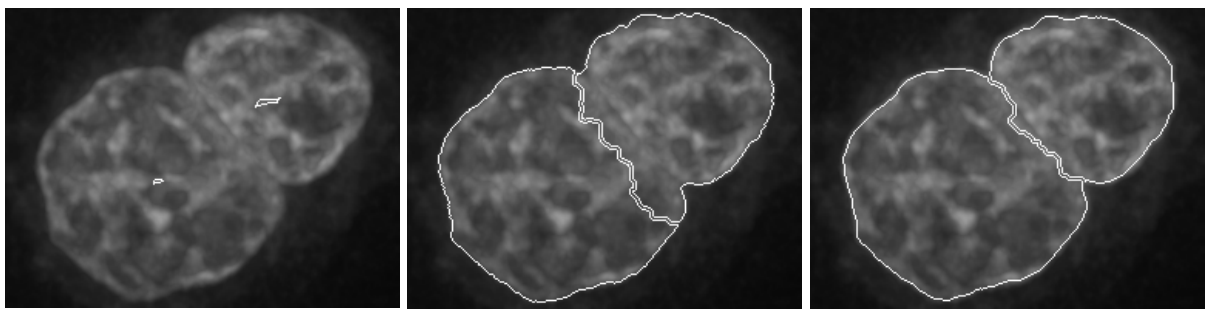

Fig. 4. Cluster separation. Left: The original image containing initial interface. Centre: The result when a constant inflation force $c=1.0$ is applied. Right: The result when a position-dependent inflation force is applied.

according to the properties of specific image data. It is also important to notice that the computational time of the second phase mainly depends on the number and shape of clusters in the image, since the isolated nuclei are not further processed in this phase. Regarding the images of HL60 cell nuclei, the first phase of our approach was not used due to a good quality of image data. Instead, a low-pass filtering followed by the Otsu thresholding were applied to obtain the foreground mask. Subsequently, the cluster separation was performed using the second phase of our method. Some examples of the final segmentation are illustrated in Fig. 5. To evaluate an accuracy of the proposed method, a measure Acc defined as a product of sensitivity (Sens) and specificity (Spec) was applied. A manual segmentation done by an expert was considered as a ground truth. The product was computed for each nucleus and averaged over all images of a cell line. The results are listed in Table 1 .

Our method, as it was described in Sect.4, is directly applicable to the segmentation of 3D images. However, its main limitation stems from the computation of the number of nuclei within a cluster and initialization of the second phase. The approach based on finding the peaks of the distance transform is not well applicable to more complex clusters that appear, for instance, in thick tissue sections. A possible solution might consist in defining the initial interface either interactively by a user or as a skeleton of each particular nucleus. The former is computationally expensive in the case of processing a huge amount of data. On the other hand, finding the skeleton of each particular nucleus is not trivial in more complex clusters. This problem will be addressed in future work.

Table 1. The parameters, average computation times and accuracy of our method. The parameter that is not applicable in a specific phase is denoted by the symbol - .

\begin{tabular}{|c|c|c|c|c|c|c|c|c|c|}
\hline Cell line & Phase & $k \quad k_{1}$ & $k_{2}$ & $\varepsilon$ & $\bar{\beta}$ & Time & Sens & Spec & $A c c$ \\
\hline \multirow[t]{2}{*}{ A549 } & 1 & 21.8 & - & 0.150 .6 & $0.16 \cdot 10^{-5}$ & $5.8 \mathrm{~s}$ & \multirow{2}{*}{\multicolumn{3}{|c|}{$96.37 \% 99.97 \% 96.34 \%$}} \\
\hline & 2 & - & 1.5 & 0.3 & $0.18 \cdot 10^{-7}$ & $3.2 \mathrm{~s}$ & & & \\
\hline \multirow[t]{2}{*}{ HL60 } & 1 & -- & - & - & - & $<1 s$ & \multirow[b]{2}{*}{$95.91 \%$} & & \\
\hline & 2 & - & 1.5 & 0.3 & $0.08 \cdot 10^{-4}$ & $2.9 \mathrm{~s}$ & & $99.95 \%$ & $95.86 \%$ \\
\hline
\end{tabular}



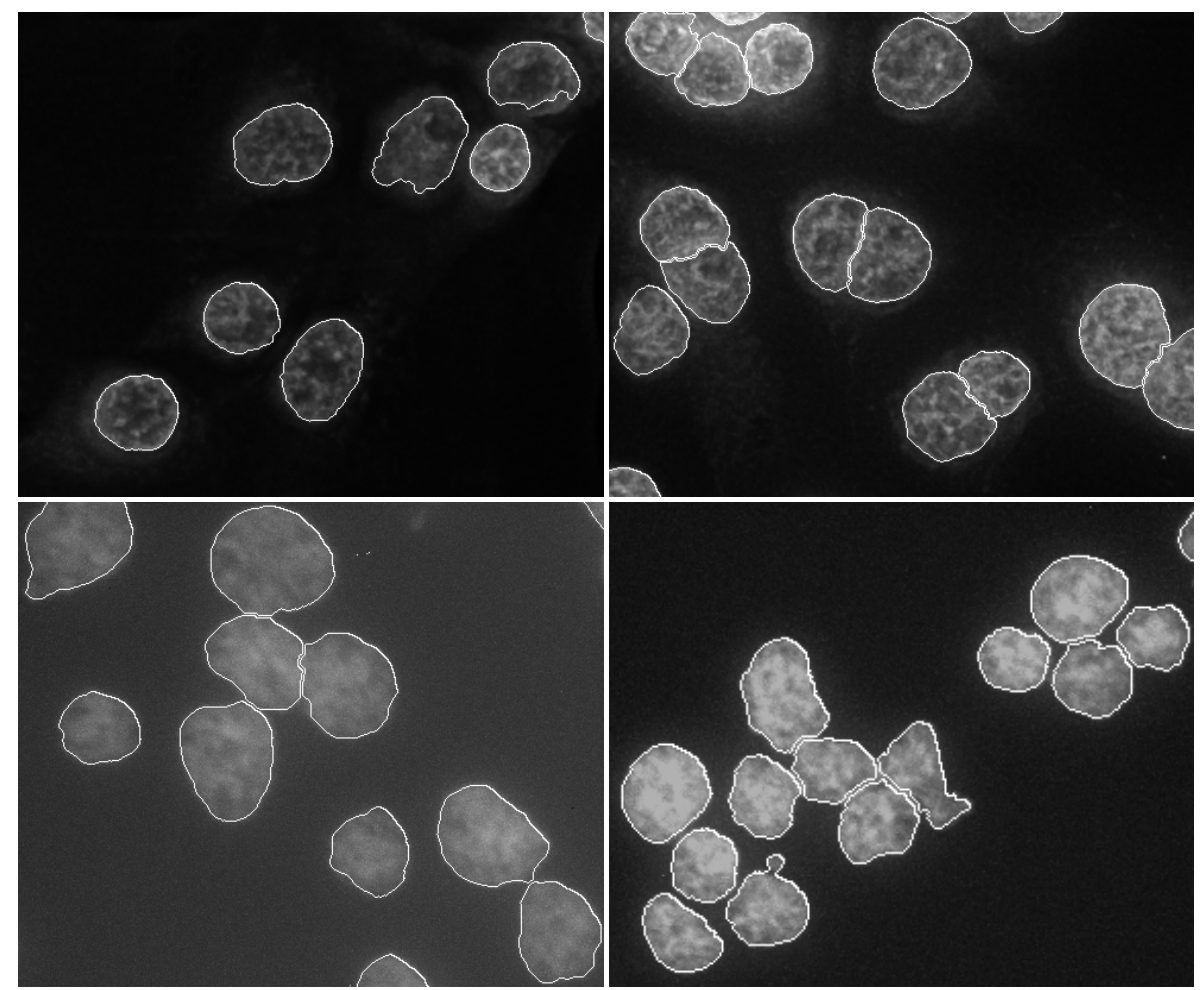

Fig. 5. Segmentation results. Upper row: The final segmentation of the A549 cell nuclei. Lower row: The final segmentation of the HL60 cell nuclei.

\section{Conclusion}

In this paper, we have presented a novel approach to the cell nucleus segmentation in fluorescence microscopy demonstrated on examples of images of a lung cancer cell line A549 as well as promyelocytic leukemia cell line HL60. The proposed method exploits the level set framework and works in two phases. In the first phase, the image foreground is separated from the background using a fast level set-like algorithm by Nilsson and Heyden. A binary mask of isolated cell nuclei as well as their clusters is obtained as a result of the first phase. A fast topology-preserving level set-like algorithm by Maška and Matula is applied in the second phase to delineate individual cell nuclei within the clusters. Our results show that the method succeeds in delineating each cell nucleus correctly in almost all cases. Furthermore, the proposed method can be reasonably used in near real-time applications due to its low computational time demands. A formal quantitative evaluation involving, in particular, the comparison of our approach with watershed-based as well as graph-cut-based methods on both real and simulated image data will be addressed in future work. We also intend to adapt the method to more complex clusters that appear in thick tissue sections. 
Acknowledgments. This work has been supported by the Ministry of Education of the Czech Republic (Projects No. MSM-0021622419, No. LC535 and No. 2B06052). COS, AMB, and IFG were supported by the Marie Curie IRG Program (grant number MIRG CT-2005-028342), and by the Spanish Ministry of Science and Education, under grant MCYT TEC 2005-04732 and the Ramon y Cajal Fellowship Program.

\section{References}

1. Nilsson, B., Heyden, A.: A fast algorithm for level set-like active contours. Pattern Recognition Letters 24(9-10), 1331-1337 (2003)

2. Maška, M., Matula, P.: A fast level set-like algorithm with topology preserving constraint. In: CAIP 2009 (March 2009) (submitted)

3. Netten, H., Young, I.T., van Vliet, L.J., Tanke, H.J., Vrolijk, H., Sloos, W.C.R.: Fish and chips: Automation of fluorescent dot counting in interphase cell nuclei. Cytometry 28(1), 1-10 (1997)

4. Gué, M., Messaoudi, C., Sun, J.S., Boudier, T.: Smart 3D-fish: Automation of distance analysis in nuclei of interphase cells by image processing. Cytometry 67(1), 18-26 (2005)

5. Malpica, N., Ortiz de Solórzano, C., Vaquero, J.J., Santos, A., Vallcorba, I., GarcíaSagredo, J.M., del Pozo, F.: Applying watershed algorithms to the segmentation of clustered nuclei. Cytometry 28(4), 289-297 (1997)

6. Wählby, C., Sintorn, I.M., Erlandsson, F., Borgefors, G., Bengtsson, E.: Combining intensity, edge and shape information for 2D and 3D segmentation of cell nuclei in tissue sections. Journal of Microscopy 215(1), 67-76 (2004)

7. Ortiz de Solórzano, C., Malladi, R., Leliévre, S.A., Lockett, S.J.: Segmentation of nuclei and cells using membrane related protein markers. Journal of Microscopy 201(3), 404-415 (2001)

8. Caselles, V., Kimmel, R., Sapiro, G.: Geodesic active contours. International Journal of Computer Vision 22(1), 61-79 (1997)

9. Chopp, D.: Computing minimal surfaces via level set curvature flow. Journal of Computational Physics 106(1), 77-91 (1993)

10. Sethian, J.A.: A fast marching level set method for monotonically advancing fronts. Proceedings of the National Academy of Sciences 93(4), 1591-1595 (1996)

11. Shi, Y., Karl, W.C.: A real-time algorithm for the approximation of level-set-based curve evolution. IEEE Transactions on Image Processing 17(5), 645-656 (2008)

12. Caselles, V., Catté, F., Coll, T., Dibos, F.: A geometric model for active contours in image processing. Numerische Mathematik 66(1), 1-31 (1993)

13. Kass, M., Witkin, A., Terzopoulos, D.: Snakes: Active contour models. International Journal of Computer Vision 1(4), 321-331 (1987)

14. Osher, S., Fedkiw, R.: Level Set Methods and Dynamic Implicit Surfaces. Springer, New York (2003)

15. Goldenberg, R., Kimmel, R., Rivlin, E., Rudzsky, M.: Fast geodesic active contours. IEEE Transactions on Image Processing 10(10), 1467-1475 (2001)

16. Kühne, G., Weickert, J., Beier, M., Effelsberg, W.: Fast implicit active contour models. In: Van Gool, L. (ed.) DAGM 2002. LNCS, vol. 2449, pp. 133-140. Springer, Heidelberg (2002)

17. Whitaker, R.T.: A level-set approach to $3 \mathrm{D}$ reconstruction from range data. International Journal of Computer Vision 29(3), 203-231 (1998)

18. Deng, J., Tsui, H.T.: A fast level set method for segmentation of low contrast noisy biomedical images. Pattern Recognition Letters 23(1-3), 161-169 (2002) 